\title{
Use of Femoral Nerve Blocks to Manage Hip Fracture Pain among Older Adults in the Emergency Department: A Systematic Review
}

\author{
Madison Riddell*; Maria Ospina, $\mathrm{PhD}^{\dagger}$; Jayna M. Holroyd-Leduc, $\mathrm{MD}^{*{ }^{\dagger \ddagger}}$
}

\section{ABSTRACT}

Objective: Hip fractures are a common source of acute pain amongst the frail elderly. One potential technique to adequately manage pain in this population is the femoral nerve block. The objective of this systematic review was to provide updated evidence for the use of femoral nerve blocks as a pain management technique for older hip fracture patients in the emergency department (ED).

Data Sources: Searches of Medline, EMBASE, and the Cochrane Central Register of Controlled Trials were conducted between December 2010 and May 2014. The reference list of a previous systematic review was also searched.

Study Selection: We included randomized control trials examining the use of femoral nerve blocks in the ED among older adults (65 years of age or older) with acute hip fracture. Data Extraction: Among 93 citations reviewed, seven trials were included. Four studies employed a single femoral nerve block, while three studies employed continuous (catheterplaced) femoral blocks. All but one of the studies were found to have a high risk of bias.

Data Synthesis: All studies reported reductions in pain intensity with femoral nerve blocks. All but one study reported decreased rescue analgesia requirements. There were no adverse effects found to be associated with the femoral block procedure; rather, two studies found a decreased risk of adverse events such as respiratory and cardiac complications.

Conclusions: Femoral nerve blocks appear to have benefits both in terms of decreasing the pain experienced by older patients, as well as limiting the amount of systemic opioids administered to this population.

\section{RÉSUMÉ}

Objectif: Les fractures de la hanche sont une cause fréquente de douleur aiguë chez les personnes âgées fragiles. L'un des moyens possibles de soulager efficacement la douleur dans ce groupe de personnes est le bloc fémoral. La revue systématique visait à recueillir des données probantes à jour sur le bloc fémoral comme moyen de soulagement de la douleur causée par les fractures de la hanche, chez les personnes âgées, au service des urgences (SU).

Sources de données: Des recherches ont été menées dans les bases de données Medline, EMBASE et Cochrane Central Register of Controlled Trials, entre décembre 2010 et mai 2014. La liste de références bibliographiques d'une revue systématique antérieure a aussi fait l'objet de recherches.

Sélection des études: Ont été sélectionnés des essais comparatifs, à répartition aléatoire, de blocs fémoraux, au $\mathrm{SU}$, chez des personnes âgées (65 ans et plus) traitées pour une fracture de la hanche.

Extraction des données: Après l'examen de 93 citations, 7 essais ont été retenus. Dans quatre d'entre eux, on avait effectué un seul bloc fémoral, tandis que dans les trois autres on avait effectué un bloc fémoral continu (à l'aide d'un cathéter). Toutes les études, à l'exception d'une seule, comportaient un risque élevé de biais.

Synthèse des données: Dans toutes les études, les auteurs ont fait état d'une diminution de l'intensité de la douleur produite par les blocs fémoraux et, sauf dans une, d'une diminution des besoins d'analgésie d'appoint. Aucun effet indésirable n'a été associé au bloc fémoral; au contraire, les auteurs ont même constaté, dans deux études, une diminution du risque d'événement indésirable tel que des complications cardiaques ou respiratoires.

Conclusions: Les blocs fémoraux semblent avantageux tant sur le plan du soulagement de la douleur chez les personnes âgées que sur celui de l'administration d'opioïdes à action générale, dans ce segment de la population.

Keywords: Hip Fracture, Femoral Nerve Blocks, Pain, Elderly

\section{INTRODUCTION}

Hip fractures are a common presentation to the emergency department (ED). Every year approximately

From the *Department of Medicine, University of Calgary, Alberta; †Alberta Health Services, Alberta; and $\ddagger$ Department of Community Health Sciences, University of Calgary, Alberta.

Correspondence to: Dr. Jayna M. Holroyd-Leduc, Foothills Hospital, $11^{\text {th }}$ Floor South Tower Room 1103, 1403-29 Street NW, Calgary, Alberta, T2N 2T9; Email: Jayna.holroyd-leduc@albertahealthservices.ca 
35,000 hip fractures occur in Canada, ${ }^{1}$ typically in older adults with poor baseline functional status and a high degree of cognitive impairment. ${ }^{2}$ As such, hip fractures are substantial contributors to pain and related morbidity within the older patient population. Morbidities include delirium, which occurs postoperatively in $15 \%$ to $53 \%$ of surgical patients over the age of $65 .{ }^{3}$ In terms of pain, hip fracture has been associated with substantial pain in both the pre- and postoperative periods. ${ }^{4}$ Adequate pain management interventions are essential to ensure that patients are not only comfortable during their hospital stay, but able to recover and return to their previous functional and cognitive health status following treatment.

Standard care to manage hip fracture pain involves the administration of systemic analgesia, typically intravenous, intramuscular, or oral opioids. Sedation and delirium are common side effects of systemic opioids when given to an older population..$^{5}$ The prevalence of delirium amongst hip fracture patients has been shown to range from $13 \%$ to $61 \%^{6}$ Furthermore, the occurrence of delirium has been shown to increase the incidence of adverse outcomes post fracture, including functional decline, permanent cognitive impairment, and death. ${ }^{7-11}$ These data highlight the acute need for alternative methods of pain management in elderly patients suffering from hip fracture.

Femoral nerve block is a procedure that has the potential to address this issue. The femoral nerve block procedure involves the injection of a local anaesthetic into the femoral nerve sheath, which then travels cranially and laterally to reach the femoral and obturator nerves, the lower chords of the lumbar plexus, and the lateral cutaneous nerve of the thigh. ${ }^{12}$ More recently, physicians have begun administering femoral nerve blocks using an ultrasound-guided technique. Ultrasound guidance allows for visualized regional anaesthesia and eliminates many of the risks associated with the traditional "blind" or nerve stimulator-guided procedure. A comparative effectiveness systematic review of the literature from 1990-2010 on pain management interventions for hip fractures demonstrated the potential feasibility and effective pain control offered by femoral nerve blocks in ED settings. ${ }^{13}$ Early studies have shown that femoral nerve blocks for hip fracture can reduce the quantity of traditional analgesia required, thereby potentially decreasing the rates of delirium. Despite potential evidence of benefit, femoral nerve blocks are not currently used in standard practice to manage hip fracture pain within many Canadian EDs, highlighting a potential knowledge-practice gap.

The objective of this systematic literature review was to examine the increasing body of research around femoral nerve blocks used to address acute hip fracture pain in the ED. Specifically, our aim was to determine whether evidence from more recent randomized controlled trials (RCTs) supports the routine implementation of femoral nerve blocks within the ED.

\section{METHODS}

\section{PICO (Population; Intervention; Comparison; Outcomes) question}

Does the use of femoral nerves blocks in the ED (preoperative) reduce acute pain, use of additional pain medications, and rates of delirium, and improve the functional status postoperatively of older adults (older than 65 years of age) with acute hip fracture as compared to placebo or standard care? (See Appendix 1)

\section{Literature search strategy}

Searches of Medline, EMBASE, and the Cochrane Central Register of Controlled Trials were conducted, with the assistance of an information specialist at the University of Calgary, to identify articles published between December 2010 and May 2014 inclusive. This time period was chosen to serve as an update to the previous systematic review performed on the topic, which included the period January 1990 to December 2010. ${ }^{13}$ The search strategies included the following keywords in combination with relevant subject headings: hip fracture, femoral neck fracture, intertrochanteric fracture, pain, therapy, anaesthesia, analgesia, frail elderly, older patients, delirium, acute confusion, cognitive impairment, confusion, femoral block, and peripheral nerve block. Further details of the search are available from the authors upon request.

A search of the reference list of the 2010 systematic review was also conducted. The search strategy of the previous 2010 review was undertaken by a technical panel of experts from multiple fields and followed a protocol dictated in the Agency for Healthcare Research and Quality Methods Guide for Effectiveness and Comparative Effectiveness Reviews. Independent reviewers performed a search of electronic databases and incorporated search strategies from reviews on similar topics. The methods 
employed by this previous review were deemed to be of high enough quality to not merit confirmation review.

\section{Study selection}

Included in this update were RCTs instituting the use of femoral nerve blocks in ED settings to treat acute hip fracture pain in patients 65 years of age or older that reported clinically measured outcomes, such as pain and analgesic consumption. The over 65 patient population was selected, as this group is at higher risk for both acute hip fractures and for adverse outcomes associated with opioid treatment. All techniques and methods of femoral nerve block administration, including those that were guided by ultrasound or peripheral nerve stimulator, were considered for inclusion in this review. Other types of regional anaesthesia that may be relevant to hip fracture pain management, such as the fascia iliaca compartment block, were excluded. This particular exclusion criterion was informed by the results of the 2010 pain management interventions for hip fracture systematic review. This past review found that there were statistically significant results in favour of the femoral nerve block, but not the fascia iliaca block.

Titles and abstracts generated from the search strategies were screened by two independent reviewers to identify potentially relevant articles. The full text of articles deemed relevant, and those whose abstracts and titles provided insufficient information, were retrieved for further inspection by two independent reviewers who determined study eligibility. Disagreements were resolved by discussion, and when necessary, a third reviewer.

\section{Risk of bias assessment}

Included trials were evaluated using the Cochrane Collaboration's Risk of Bias tool, ${ }^{14}$ which includes an evaluation of random sequence generation, allocation concealment, blinding of participants and personnel, blinding of outcome assessment, incomplete outcome data, selective reporting, and other sources of bias. Two independent reviewers assessed risk of bias of individual studies, and disagreements were resolved by consensus or third party adjudication.

\section{Data extraction and analysis}

Two reviewers independently extracted data from the included studies using a standardized data abstraction form.
The following information was obtained from individual studies: design, country, study population and size, clinical setting, characteristics of the intervention and control groups, outcomes, results, and study conclusions. A meta-analysis was not conducted due to the clinical heterogeneity across the studies. Clinical heterogeneity was encountered in the types of femoral nerve block procedures used, such as catheter placement versus single nerve block techniques. Heterogeneity was also present in terms of the methods used to assess pain, for instance verbal numerical rating scales versus visual analogue scales. Instead, a narrative synthesis of effectiveness outcomes across the studies is presented.

\section{RESULTS}

\section{Study characteristics}

The electronic search strategy identified 89 citations. Four additional citations were identified from the reference list of a previous 2010 systematic review. After screening of titles and abstracts, 13 studies were selected as potentially relevant, of which seven RCTs $^{15-21}$ satisfied the eligibility criteria for inclusion (Figure 1). Among the included studies, four studies ${ }^{15-18}$ employed a single femoral nerve block intervention, while three studies ${ }^{19-21}$ employed continuous (catheterplaced) femoral nerve blocks (Table 1). Studies were conducted in the United Kingdom (UK), ${ }^{15,16}$ the United States (US), ${ }^{17,18}$ France, ${ }^{19}$ Austria, ${ }^{20}$ and Ireland. ${ }^{21}$ As shown in Table 2, all but one of the studies ${ }^{18}$ were found to have a high risk of bias.

All the studies reported reductions in pain intensity, both with movement and at rest, in those receiving the femoral nerve block (Table 1). In addition, all but one study ${ }^{17}$ reported that patients treated with femoral nerve blocks consumed significantly less total rescue analgesia (morphine or morphine equivalent) compared to controls. There were no adverse effects found to be associated with the femoral nerve block procedure, and two studies found a decreased risk of adverse events such as respiratory ${ }^{16}$ and cardiac $^{20}$ complications.

\section{Single femoral nerve block intervention}

Four of the reviewed studies ${ }^{15-18}$ focused on single femoral nerve blocks as an intervention. One RCT ${ }^{15}$ assessed the effectiveness of single femoral nerve blocks compared to intravenous morphine in a medium-sized 


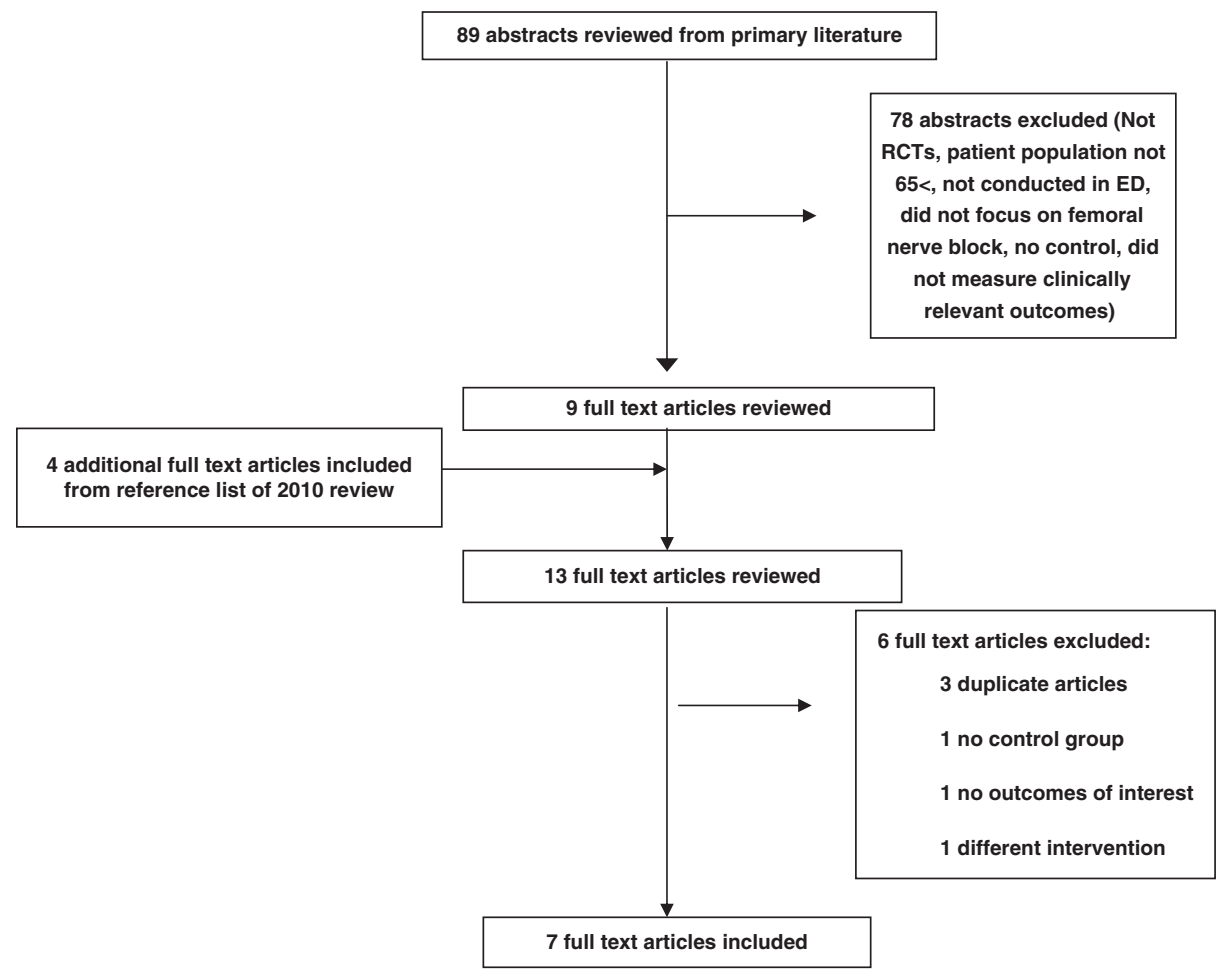

Figure 1. Literature search results for the use of femoral nerve blocks to manage hip fracture pain among the elderly patient population.

district hospital in the UK. The primary outcome of the study was pain intensity measured using a verbal numerical pain scale from zero to three. Results revealed that the group that received the femoral nerve block reported pain scores that were on average 0.78 points $(95 \%$ confidence interval $[\mathrm{CI}]: 1.02,0.54)$ lower than those of the control group. Additionally, the femoral nerve block group consumed an average of 0.68 (95\% CI: 1.23, 0.12) fewer milligrams of morphine per hour than the control group.

An $\mathrm{RCT}^{16}$ conducted among patients in the UK with extracapsular fractures of the femoral neck measured pain scores via a zero to ten visual analogue scale. Compared to the control group that received intramuscular opiates, patients who received the femoral nerve block had significantly lower scores in pain intensity 15 minutes after the injection (1.6 points lower; $p<0.05)$, and two hours after the injection (2.2 points lower; $p<0.01$ ). Furthermore, this study revealed a significant decrease $(p<0.05)$ in the amount of intramuscular opiates required by the femoral nerve block group. Additionally, the femoral nerve block group had significantly fewer postoperative respiratory complications $(p<0.05)$.
A small $\mathrm{RCT}^{17}$ (14 patients) conducted in the US found that a group who received a femoral nerve block in the ED had a statistically significant reduction in pain one hour after the procedure was performed, as measured via an 11-point verbal numerical rating scale. The mean pain intensity reported by the femoral nerve block group was 2.7 , while the patient group receiving standard care reported a mean pain intensity score of $6.1(p<0.04)$. The study found similar administration of morphine equivalents between the two groups. Although not significant, reductions in pain were reported by the femoral nerve block group at four and 24 hours after the procedure.

The most recent $\mathrm{RCT}^{18}$ assessed the effectiveness of single femoral nerve block compared to standard care in a large urban academic ED in the US. Pain intensity was measured using an 11-point numerical rating scale. The study reported a significant reduction in pain in the femoral nerve block group four hours after the procedure $(p<0.001)$ compared to the group receiving standard care alone. The femoral nerve block group was also found to have experienced significantly greater levels of pain relief, as the summed pain intensity differences reported by the femoral nerve block group 


\begin{tabular}{|c|c|c|c|c|c|c|}
\hline Ref \# & Location & $\mathrm{ROB}$ & $\mathrm{N}$ & Block & Control type & Results \\
\hline 15 & UK & $\mathrm{H}$ & 50 & Single & $\begin{array}{l}\text { Standard care } \\
\text { (IV morphine) }\end{array}$ & $\begin{array}{l}\text { - Mean difference in pain (0-3 VRS) was } 0.78 \text { points lower in } \\
\text { - FNB group. } \\
\text { - FNB patients required less morphine per hour than control } \\
\text { - } \\
\text { No adients (mean difference was } 0.68 \mathrm{mg} / \mathrm{h} \text { lower).* } \\
\end{array}$ \\
\hline 16 & UK & $\mathrm{H}$ & 50 & Single & $\begin{array}{r}\text { Standard care } \\
\text { (IM opiates) }\end{array}$ & $\begin{array}{l}\text { - Reduction in pain in the FNB patients at } 15 \text { minutes* }, 2 \text { hours* } \\
\text { and } 8 \text { hours after the injection (i.e., at } 2 \text { hours } 3.7 \text { points lower } \\
\text { in FNB group vs } 1.6 \text { points lower in control group). } \\
\text { - } \text { Reduction in requirement for IM opiates in the FNB group } \\
\text { (12 vs } 35) .^{*} \\
\text { - Decrease in the incidence of postoperative respiratory } \\
\text { depression.* }\end{array}$ \\
\hline 17 & US & $\mathrm{H}$ & 14 & Single & $\begin{array}{l}\text { Standard care } \\
\text { (opioids) }\end{array}$ & $\begin{array}{l}\text { - Pain intensity was lower in the FNB group after } 1 \text { hour as } \\
\text { measured by an 11-point NRS ( } 2.7 \text { for FNB vs } 6.1 \text { for SC).* } \\
\text { - Total morphine equivalents were similar. } \\
\text { - }\end{array}$ \\
\hline 18 & US & L & 36 & Single & $\begin{array}{l}\text { Placebo (sham } \\
\text { injection) plus } \\
\text { standard care } \\
\text { (morphine) }\end{array}$ & $\begin{array}{l}\text { - Lower pain intensity in the FNB group as measured by } \\
\text { 11-point NRS at } 4 \text { hours }(11(-4-44) \text { vs } 4(-7-11)) \text { (FNB vs } \\
\text { control).* } \\
\text { - Patients in the FNB group received less IV morphine than the } \\
\text { control group (0 mg (0-6) vs } 5.0 \mathrm{mg}(0-21)) \text { (FNB vs control).* } \\
\text { - No adverse events. }\end{array}$ \\
\hline 19 & France & $\mathrm{H}$ & 30 & Continuous & $\begin{array}{c}\text { Standard care } \\
\text { (intravenous } \\
\text { morphine) }\end{array}$ & $\begin{array}{l}\text { - Pain scores on a } 0 \text { to } 10 \text { VRS were reduced in the FNB group: } \\
\text { mean difference: } 2.1 \pm 2.1 \text { vs } 6.5 \pm 3.5 \text { for SC. } \\
\text { - Amount of morphine/morphine equivalent not recorded. } \\
\text { - No adverse events. }\end{array}$ \\
\hline 20 & Austria & $\mathrm{H}$ & 20 & Continuous & $\begin{array}{l}\text { Standard care } \\
\text { (opiods) }\end{array}$ & $\begin{array}{l}\text { - Lowered pain intensity in the FNB group as measured by VAS } \\
\text { both in motion and at rest: mean difference: }-8.3 \text { vs }-7.5 \\
\text { (FNB vs control)* and mean difference: }-6.0 \text { vs }-5.9 \text { (FNB vs } \\
\text { control).* } \\
\text { - } \text { FNB group required less analgesia: i.e., paracetamol } \\
\text { consumption }+0.1 \mathrm{mg} / \mathrm{d} \text { vs }+1 \mathrm{mg} / \mathrm{d} \text { (FNB vs control). }{ }^{*} \\
\text { - } \text { Increased cardiovascular stability in FNB group as determined } \\
\text { by serum troponin levels.* }\end{array}$ \\
\hline 21 & Ireland & $\mathrm{H}$ & 24 & Continuous & $\begin{array}{l}\text { Standard care } \\
\text { (opiods) }\end{array}$ & $\begin{array}{l}\text { - Lower pain scores reported on a 0-100 VAS in the FNB group } \\
\text { than in the control group: mean difference: }-28.4 \text { vs }-13.1 \\
\text { (FNB vs control).* } \\
\text { - Lower cumulative morphine consumption over } 72 \text { hours in the } \\
\text { FNB group compared to the control group: } 4.8 \mathrm{mg} \text { vs } 20.3 \mathrm{mg} \\
\text { (FNB vs control).* } \\
\text { - No adverse events. }\end{array}$ \\
\hline
\end{tabular}

had a median of 11.0 (4 to 21.8 ) versus 4.0 ( -2 to 5.8 ) in the standard care group. Additionally, the study showed patients in the control group required significantly more intravenous morphine than patients in the femoral nerve block group $(p=0.028)$; the control group reported a median of $5 \mathrm{mg}$ of morphine $(2.0 \mathrm{mg}$ to $8.4 \mathrm{mg}$ ) versus $0 \mathrm{mg}(0 \mathrm{mg}$ to $1.5 \mathrm{mg})$ in the femoral nerve block group. 


\begin{tabular}{|c|c|c|c|c|c|c|c|}
\hline Citation identifier & \#15 & $\# 16$ & $\# 17$ & $\# 18$ & $\# 19$ & \#20 & \#21 \\
\hline Random sequence generation & Low & Low & Unclear & Low & Low & Low & Low \\
\hline Allocation concealment & Low & High & High & Low & Unclear & Low & Unclear \\
\hline Blinding of participants and personnel & High & High & High & Low & Low & Unclear & High \\
\hline Blinding of outcome assessment & Low & Low & Unclear & Low & Unclear & Unclear & Unclear \\
\hline Incomplete outcome data & Low & Unclear & Unclear & Low & Unclear & Low & Low \\
\hline Selective outcome reporting & Low & High & Low & Low & Unclear & Low & Low \\
\hline Other sources of bias & High & Unclear & High & Low & High & High & High \\
\hline Overall risk of bias & High & High & High & Low & High & High & High \\
\hline
\end{tabular}

\section{Continuous (catheter-placed) femoral nerve block intervention}

Three RCTs ${ }^{19-21}$ investigated the effectiveness of continuous (catheter-placed) femoral nerve block. One RCT $^{19}$ conducted in an ED in France used a verbal numerical rating scale between zero and 10 to assess the effect of continuous femoral nerve blocks in pain intensity outcomes of 16 hip fracture patients. A statistically significant $(p<0.001)$ difference in mean pain scores was observed between the two groups. The femoral nerve block group reported a mean pain score of 2.1 ( \pm 2.1 standard deviation [SD]), while the control group reported an average pain score of $6.5( \pm 3.5 \mathrm{SD})$.

The $\mathrm{RCT}^{20}$ conducted in Austria evaluated the analgesic effectiveness of continuous femoral nerve blocks on pain intensity, measured by a visual analog scale ranging from zero to 10 . Significant differences $(p<0.05)$ in pain intensity were observed between continuous femoral nerve block (mean score of 0.8 ) and control groups (mean score of 4.7) after one hour. Although not significant, this study revealed that patients receiving the block experienced a lower pain score on both the visual analog scale and the verbal rating scale 12 hours after the block was initiated. The study also found that consumption of additional analgesics was elevated in the control group when compared to the treatment group. Cardiovascular instability, determined by measuring patients' maximum serum troponin levels, was significantly lower in the femoral nerve block study group compared to standard care $(p<0.03)$.

The RCT ${ }^{21}$ conducted in Ireland sought to investigate the analgesic efficacy of continuous femoral nerve block administered to ED patients presenting with femoral neck fractures. Pain intensity was assessed using a visual analog scale (0-100) every six hours for 72 hours, while patients were at rest and in motion.
The study showed that, in rest conditions, patients receiving the femoral nerve block had significantly lower pain scores than those in the control group (9.5 versus $31 ; p=0.031$ ). Additionally, pain scores while in motion were lower in the femoral nerve block group at each time point up to 54 hours. For example, at six hours after the catheter was placed, the femoral nerve block group demonstrated average pain scores of 30.7 versus 67.0 in the control group. Additional outcomes evaluated in this trial were cumulative morphine consumption and patient satisfaction. The study revealed that the femoral nerve block group required less morphine over the 72-hour study period, while also reporting significantly higher patient satisfaction scores $(p=0.014)$ compared to the group receiving standard care alone.

\section{DISCUSSION}

This systematic review aimed to identify new RCT evidence regarding the effectiveness of femoral nerve blocks administered to hip fracture patients in the ED, expanding on and updating the systematic review by Abou-Setta et al. ${ }^{13}$ The findings of the systematic review presented here were consistent with the review by Abou-Setta et al.: both single and continuous femoral nerve blocks appear to have a positive impact on elderly patients presenting to the ED with acute hip fracture. The benefits include decreased pain intensity, a decrease in the amount of required rescue analgesia administered, and fewer adverse events.

Of the four studies ${ }^{15-18}$ assessing single femoral nerve block interventions, all but one ${ }^{15}$ found a significant reduction in pain intensity. All three $\mathrm{RCTs}^{19-21}$ that investigated the clinical application of continuous femoral nerve blocks showed a significant reduction in pain compared to standard care. This reduction in pain intensity may play a critical role in a patient's recovery 
process, and it has been shown in one study that pain severity postoperatively correlates with health-related quality of life. ${ }^{22}$ Additionally, pain is one of the most common reasons for hospital readmissions. ${ }^{23}$

All review studies but one ${ }^{17}$ reported a decreased requirement for rescue analgesia among patients receiving femoral nerve blocks. By reducing the amount of systemic opioids required by older patients with acute hip fractures, femoral nerve blocks may help to decrease the incidence of delirium. Delirium is a known side effect of systemic opioids among the elderly. ${ }^{24}$ Additional side effects or adverse events that may also be decreased with a reduction in systemic narcotic use include nausea, constipation, pruritus, respiratory depression, cardiovascular instability, and death. ${ }^{25}$ The studies included in this review showed no adverse events resulting from administration of femoral nerve blocks, further supporting its integration into routine clinical practice for pain management among hip fracture patients.

Although it may be inferred from the current review findings that delirium rates would decrease with the use of femoral nerve blocks, this was not a specific outcome in any of the included studies. Future studies should explore the impact of femoral nerve blocks on delirium rates, along with associated outcomes such as functional status.

It is important to note that various femoral nerve block techniques were included in this review. Two studies ${ }^{15,16}$ used the loss of resistance method, three studies ${ }^{17,19,21}$ used a peripheral nerve stimulator, and two studies ${ }^{18,20}$ used ultrasound technology to employ the nerve block. Regardless of the method of nerve block administration, significant benefits were reported. However, other studies suggest that ultrasound guidance may increase the efficacy and feasibility of this procedure in the $\mathrm{ED}^{26}$ and may be superior to other nerve block techniques in regard to onset of action and amount of anaesthetic required. ${ }^{27}$

Both catheter-placed and single femoral nerve block procedures were included in this review. Although both techniques showed benefits in terms of pain reduction, there are important differences between the two techniques that may have important clinical implications. Within the Canadian context where surgical wait time benchmarks for hip fracture repair are within 48 hours, ${ }^{28}$ the continuous femoral nerve block, which extends the duration of analgesia, may be important. However, the continuous nerve block is a more technically complex procedure.
Despite the potential benefit of this procedure, femoral nerve blocks have yet to be instituted as a routine pain management technique in Canadian EDs. Potential barriers to implementing femoral nerve block interventions need to be explored further. One potential barrier appears to be the belief that administering a femoral nerve block requires specialized training and is usually performed by anaesthesiologists. ${ }^{29}$ However, in our review, one of the studies found that the procedure required minimal instruction and could be performed by junior staff, ${ }^{16}$ and multiple studies ${ }^{15,17,18}$ reported relatively brief training required to administer the nerve blocks. Another perceived barrier may be the longer time required to perform a femoral nerve block, as compared to traditional methods of pain management. However, two published studies ${ }^{30,31}$ found that nerve blocks increased efficiency by reducing the total operation time, as well as the incidence of readmission due to pain. In order to effectively implement femoral nerve blocks into routine $\mathrm{ED}$ care, the barriers to use need to be explored further, and then implementation interventions that specifically address these identified barriers need to be developed and evaluated.

The strengths of this systematic review pertain to its rigor in searching the literature, the criteria-based selection of relevant evidence, the rigorous appraisal of study validity, and the evidence-based inferences. Although the identification of a relatively small number of studies reporting comparable outcome measures precluded a visual assessment of potential publication bias via funnel plots, the comprehensive search strategy is likely to have identified most of the available RCT literature on the effectiveness of femoral nerve blocks to manage hip fractures in the frail older patients visiting the ED.

\section{CONCLUSIONS}

This review helps to consolidate current knowledge that femoral nerve blocks appear to be an effective option to manage acute pain following hip fractures in the frail older population. This procedure decreases the level of pain intensity experienced by the older patient, reduces the amount of systemic analgesia required, and appears to have fewer adverse events associated with it. Implementation of femoral nerve blocks into routine clinical practice could improve the quality of care provided to the frail older patient experiencing a hip fracture. 
Competing Interests: Ms. Riddell received funding from a TVN (Technology Evaluation in the Elderly) NCE Summer Studentship to complete this review at the University of Calgary. Dr. Holroyd-Leduc received funding from Alberta Health Services in her role as the Scientific Director of the Seniors Health Strategic Clinical Network. Dr. Ospina was funded by Alberta Health Services in her role as the Assistant Scientific Director of the Emergency Strategic Clinical Network.

\section{SUPPLEMENTARY MATERIAL}

To view supplementary material for this article, please visit http://dx.doi.org/10.1017/cem.2015.94

\section{REFERENCES}

1. Freter S, Koller K. Hip fractures and Alzheimer's Disease. The Canadian Review of Alzheimer's Disease and Other Dementias 2008;11:15-20.

2. Gerson LW, Emond JA, Camargo C. US emergency department visits for hip fracture, 1992-2000. Eur 7 Emerg. Med 2004;11(6):323-8.

3. Inouye SK. Delirium in older persons. $N$ Engl $7 \mathrm{Med}$ 2006;354(11):1157-65.

4. National Institute for Health and Clinical Care Excellence. Hip fracture: the Management of Hip fracture in Adults. London: NICE; 2011.

5. Holroyd-Leduc J, Khandwala F, Sink KM. Delirium: How can it be best prevented and managed in older patients in hospital? CMA7 2010;182(5):465-70.

6. Edlund A, Lundstrom M, Ludstrom G, et al. Clinical profile of delirium in patients treated for femoral neck fractures. Dement Geriatr Cogn Disord 1999;10(5):325-9.

7. Francis J, Kapoor WN. Prognosis after hospital discharge of older medical patients with delirium. $7 \mathrm{Am}$ Geriatr Soc 1992;40(6):601-6.

8. Gustafson Y, Berggren D, Brannstrom B, et al. Acute confusional states in elderly patients treated for femoral neck fracture. 7 Am Geriatr Soc 1988;36(6):525-30.

9. Inouye SK, Rushing JT, Foreman MD, et al. Does delirium contribute to poor hospital outcomes? A three-site epidemiologic study. 7 Gen Intern Med 1998;13(4):234-42.

10. O'Keeffe S, Lavan J. The prognostic significance of delirium in older hospital patients. $\mathcal{F}$ Am Geriatr Soc 1997;45(2):174-8.

11. Marcantonio ER, Flacker JM, Michaels M, et al. Delirium is independently associated with poor functional recovery after hip fracture. 7 Am Geriatr Soc 2000;48(6):618-24.

12. Fournier R, Van Gessel E, Gaggero G, et al. Postoperative analgesia with "3-in-1" femoral nerve block after prosthetic hip surgery. Can 7 Anaesth 1998;45(1):34-8.

13. Abou-Setta AM, Beaupre LA, Rashiq S, et al. Comparative effectiveness of pain management interventions for hip fracture: a systematic review. Ann Intern Med 2011;155(4): 234-45.

14. Higgins JPT, Altman DG, Gotzsche PC, et al. The Cochrane Collaboration's tool for assessing risk of bias in randomized trials. BM7 2011;343:d5928.
15. Fletcher AK, Rigby AS, Heyes FL. Three-in-one femoral nerve block as analgesia for fractured neck of femur in the Emergency Department: A randomized, control trial. Ann Emerg Med 2003;41(2):227-33.

16. Haddad FS, Williams RL. Femoral nerve block in extracapsular femoral neck fractures. 7 Bone foint Surg Br 1995; 77(6):922-3.

17. Henderson K, Akhtar S, Sandoval M, et al. Femoral nerve block for pain management of hip fractures in the emergency department: preliminary results of a randomized, controlled trial. Ann Emerg Med 2008;52(4):S164.

18. Beaudoin FL, Haran JP, Liebmann O. A comparison of ultrasound-guided three-in-one femoral nerve block versus parenteral opiods alone for analgesia in emergency department patients with hip fractures: A randomized controlled trial. Acad Emerg Med 2013;20(6):584-91.

19. Murgue D, Ehret B, Massacrier-Imbert S, et al. Equimolar nitrous oxide/oxygen combined with femoral nerve block for emergency analgesia of femoral neck fractures. 7 Eur Urg 2006;19(1):9-14.

20. Luger TJ, Kammerlander C, Benz M, et al. Peridural anesthesia or ultrasound-guided continuous 3-in-1 block: which is indicated for analgesia in very elderly patients with hip fracture in the emergency department? Geriatr Orthop Surg Rebab 2013;3(3):121-8.

21. Szucs S, Iohom G, O’Donnel B, et al. Analgesic efficacy of continuous femoral nerve block commenced prior to operative fixation of fractured neck of femur. Perioper Med (Lond) 2012;1:4. doi: 10.1186/2047-0525-1-4.

22. Wu CK, Naqibuddin M, Rowlingson AJ, et al. The effect of pain on health-related quality of life in the immediate postoperative period. Anesth Analg 2003;97(4):1078-85.

23. Sinatra R. Causes and consequences of inadequate management of acute pain. Pain Med 2010;11(12):1859-71.

24. Pasero C, McCaffery M. Reluctance to order opiods in elders. Am 7 Nurs 1997;97(9):20-3.

25. Chau DL, Walker V, Pai L, et al. Opiates and elderly: use and side effects. Clin Interv Aging 2008;3(2):273-8.

26. Beaudoin FL, Nagdev A, Merchant RC, et al. Ultrasoundguided femoral nerve blocks in elderly patients with hip fractures. Am 7 Emerg Med 2010;28(1):76-81.

27. Marhofer P, Schrogendorfer K, Wallner T, et al. Ultrasonographic guidance reduces the amount of local anesthetic for 3-in-1 blocks. Reg Anesth Pain Med 1998;23(6):584-8.

28. Canadian Institute for Health Information. Wait Times for Priority Procedures in Canada, 2014; March 2014. Available at: https://secure.cihi.ca/free_products/2014_WaitTimesAiB_ EN.pdf (accessed: March 20, 2015).

29. Practice guideline for acute pain management in the perioperative setting: An updated report by the American Society of Anesthesiologists task force on acute pain management. Anesthesiology 2004;100(6):1573-81.

30. Armstrong KP, Cherry RA. Brachial plexus anesthesia compared to general anesthesia when a block room is available. Can $\mathcal{F}$ Anaesth 2004;51(1):41-4.

31. Gonano C, Kettner SC, Ernstbrunner M, et al. Comparison of economical aspects of interscalene brachial plexus blockade and general anaesthesia for arthroscopic shoulder surgery. Br 7 Anaesth 2009;103(3):428-33. 